\title{
Active laser beam correction using continuous-surface deformable mirrors: algorithm and laboratory tests
}

\author{
Héctor González-Núñez ${ }^{1, S^{*}}$, Clémentine Béchet ${ }^{1}$, Boris Ayancán ${ }^{1}$, Andrés Guesalaga ${ }^{1}$ \\ 1. Pontificia Universidad Católica de Chile, 4860 Vicuña Mackenna, Santiago, Chile \\ (*) E-mail: hegonzalez@uc.cl S: SEDOPTICA member \\ Received: 27/10/2016 Accepted: 02/03/2018 \\ DOI: $10.7149 /$ OPA.51.1.49017
}

\begin{abstract}
:
We propose an optically efficient active beam-shaping system which consists of two continuoussurface deformable mirrors correcting for both phase and amplitude distortions to improve the beam quality of a laser. We present the results obtained with simulations and we validate next the technique using an experimental setup with two Boston micromachines MEMS deformable mirrors of 12 x 12 actuators each.
\end{abstract}

Key words: Laser beam shaping; Deformable mirrors.

\section{REFERENCES AND LINKS / REFERENCIAS Y ENLACES}

[1] W. Silfvast, Laser Fundamentals. Cambridge University Press (2004).

[2] M. Dickey, ed., Laser Beam Shaping: Theory and Techniques, 2nd ed. CRC Press (2014).

[3] C. Momma, S. Nolte, G. Kamlage, F. V. Alvensleben, and A. Tünnermann, "Beam delivery of femtosecond laser radiation by diffractive optical elements," Appl. Phys. A 67, 517-520 (1998).

[4] MJ. A. Hoffnagle, C. M. Jefferson, "Design and performance of a refractive optical system that converts a Gaussian to a flattop beam," Appl. Opt. 39, 5488-5499 (2000).

[5] F. Duerr, H. Thienpont, "Refractive laser beam shaping by means of a functional differential equation based design approach," Opt. Express 22, 8001-8011 (2014).

[6] T. Bifano, "Adaptive imaging: MEMS deformable mirrors," Nat. Photonics 5, 21-23 (2011).

[7] H. Yu, J. Park, K. Lee, J. Yoon, K. Kim, S. Lee, and Y. Park, "Recent advances in wavefront shaping techniques for biomedical applications," Curr. Appl. Phys. 15, 632-641 (2015).

[8] M. B. Roopashree, A. Vyas, B. Prasad, "A novel model of influence function: calibration of a continuous membrane deformable mirror, " ACEEE Int J Contr Syst Instrum 03, 10-14 (2012).

[9] C. Béchet, A. Guesalaga, B. Neichel, V. Fesquet, D. Guzman, "A two deformable-mirror concept to improve the laser efficiency of Gemini South MCAO (GeMS), " Proc. of the Third A04ELT Conference, 13344 (2013).

[10] C. Béchet, A. Guesalaga, B. Neichel, V. Fesquet, H. González-Núñez, S. Zúñiga, P. Escarate, D. Guzman, "Beam shaping for laser-based adaptive optics in astronomy, " Opt. Express 22, 12994-13013 (2014).

[11] R. W. Gerchberg, W. O. Saxton, "A practical algorithm for the determination of phase from image and diffraction plane pictures, " Optik 35, 237-250 (1971).

[12] O. Ripoll, V. Kettunen, H. P. Herzig, "Review of iterative fourier-transform algorithms for beam shaping applications, " Optical Engineering 43(11), 2549-2556 (2004).

[13] J. D. Schmidt, Numerical simulation of optical wave propagation with examples in MATLAB. Washington, SPIE (2010).

[14] S. Zúñiga, C. Béchet, H. González-Núñez, B. Neichel, V. Fesquet, V. Garrel, P. Escárate, M. Castro, D. Guzmán, A. Guesalaga, "Laboratory validation of a laser shaping system before guide star projection, " Proc. SPIE 9148, 91483I-10 (2014). 
[15] H. González-Núñez, C. Béchet, B. Ayancán, B, Neichel, and A. Guesalaga, "Effect of the influence function of deformable mirrors on laser beam shaping," Appl. Opt. 56, 1637-1646 (2017).

\section{Introduction}

The quality of the output beam is a critical parameter in the practical performance of a laser for a wide range of applications. Various techniques have been proposed to solve the problem of changing a given light distribution into a better-suited form $[1,2]$. Some seek to modulate the input laser beam by using static devices, such as amplitude masks or spatial filters, but this type of beam shaping represents an inefficient use of the output beam power [3]. Furthermore, the techniques that use static devices such as refractive or diffracting elements are incompatible with applications requiring adaptability to time-dependent effects [35].

Dynamic shaping generally involves the use of optical liquid-crystal-on-silicon (LCOS) or deformable mirrors (DMs) optical devices [6]. Comparing with continuous-surface DMs, LCOS and other segmented devices have lower reflectivity, lower fill factor and their segmented surface scatters light [7]. Thus, continuous-facesheet DMs are the natural choice for applications where it is mandatory to avoid power losses. However, the use of continuous-surface DMs faces two specific difficulties: (1) The mechanical coupling between actuators (because of their common attachment to the continuous membrane of the DM) characterized by the influence function [8] and (2) the need to develop phase retrieval algorithms with phase unwrappers to ensure that the deformation can be reproduced by the continuous membrane.

The beam-forming system presented in this work consists of two continuous-surface DMs along with the algorithm to control them $[9,10]$. The first mirror changes the laser beam intensity distribution and the second mirror modifies the phase to improve the propagation of the beam. If it is not necessary to propagate the beam over a long distance, the second mirror may not be necessary. The system can be used in openloop or close-loop configurations. A single wavefront sensor (WFS) is only strictly necessary to characterize the amplitude and phase of the initial beam.

The study presented in this work is focused in the experimental implementation of the proposed laser beam correction system. According to this, in Section 2 we briefly lay out the algorithm for active laser beam correction. Section 3 describes the experimental set-up for the beam-forming system implemented in the laboratory with two Boston micromachines MEMS DMs. In Section 4 we present the numerical and experimental results obtained. Finally, Section 5 contains the conclusions of this work.

\section{Algorithm for active laser beam correction.}

In 1972, Gerchberg and Saxton published the first iterative Fourier transform algorithm adapted for phase retrieval problems known as the error-reduction algorithm [11]. Since then, many iterative Fourier transform algorithms have been proposed to transform a given light distribution into a different light distribution related by a propagation function [12]. However, this family of algorithms were not suited to deal with continuous-surface elements such as the membrane of a DM [9-12].

A new near-field phase retrieval algorithm based on the error-reduction algorithm with a novel iterative unwrapper was developed by our group and published in [9] and [10]. Comparing with the classical errorreduction algorithm, the two key modifications are: (1) the propagation is performed according to Fresnel approximation (near-field propagation) [13], and (2) a specific iterative unwrapper was inserted into the iterative error-reduction algorithm modifying the phase freedom conditions $[10,12]$ to ensure that the retrieved phase can be easily reproduced by the smooth and continuous surface of the DM.

The active laser beam correction consists in determining the shape of the first DM surface to spatially redistribute an arbitrary incoming beam into a desired amplitude distribution on the surface of the second DM. Then, the second mirror modifies the phase of the field according with the desired phase specifications. Thus, the user indicates the desired amplitude and the desired phase at the output plane (placed on the surface of the second DM) and the algorithm provides, for arbitrary input fields, the shape of both DMs' surfaces. For a detailed description of the algorithm, the reader is referred to [10]. 
This algorithm was partially validated in [14] and in [15] with a single DM and only for laser beam shaping applications. In the present work, we present the fully implementation of the 2-DM concept in the laboratory and we present the first experimental results for active laser beam correction.

\section{Experimental set-up}

The main components of the experimental set-up are the two Boston micromachines MEMS deformable mirrors with $12 \times 12$ actuators each (see Fig. 1). Table 1 is a listing of the specifications of both devices.
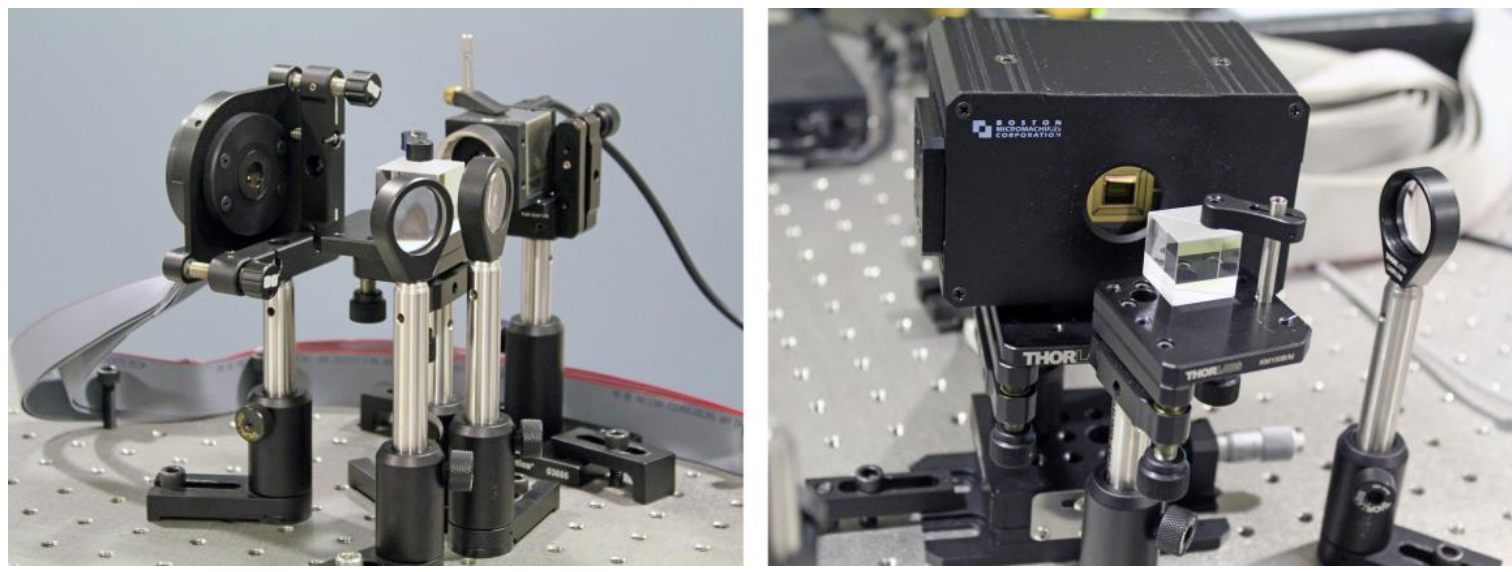

Fig.1. Images of the two Boston micromachines MEMS deformable mirrors: Left panel shows the Multi-5.5 DM and the right panel shows the Multi-3.5 DM (see Table 1).

TABLE 1. Specifications of the MEMS DMs

\begin{tabular}{|c|c|c|}
\multicolumn{1}{c}{} & Multi-3.5 (DM2) & Multi-5.5 (DM1) \\
\hline Actuator Count & $140(12 \times 12)$ & $140(12 \times 12)$ \\
Max. Stroke & $3.5 \mu \mathrm{m}$ & $5.5 \mu \mathrm{m}$ \\
Active aperture & $4.4 \mathrm{~mm}$ & $4.95 \mathrm{~mm}$ \\
Actuator pitch & $400 \mu \mathrm{m}$ & $450 \mu \mathrm{m}$ \\
Interactuator Coupling & $13 \%$ & $22 \%$ \\
\hline
\end{tabular}

MEMS DMs consist of a mirror membrane supported by an underlying actuator array. Each actuator can be individually deflected by electrostatic actuation to achieve the desired deformation. Unlike piezoelectric mirrors, electrostatic actuation ensures mirror deformation without hysteresis. The absence of hysteresis facilitates the use of the DMs in an open-loop configuration. In the context of this work, open-loop configurations are more efficient in terms of power losses since closed-loop configurations need to spend some light to sense the output field. The main disadvantage of these devices is the presence of a printed pattern on the membrane's surface [15]. Small diffractive effects associated with this pattern appear in the experimental results (see Section 4).

The experimental characterization of the beam in different planes is a crucial point. To accomplish this task, we use three Thorlabs' Shack-Hartmann WFSs. They provide accurate measurements of the wavefront shape and intensity distribution of the beam. The WFS are placed in different planes of the experimental set-up to monitor the system performance (see Figs. 2 and 3). It is important to highlight that in the final set-up only a single WFS is necessary to characterize the beam (amplitude and phase) at the input plane (see Fig. 2). 


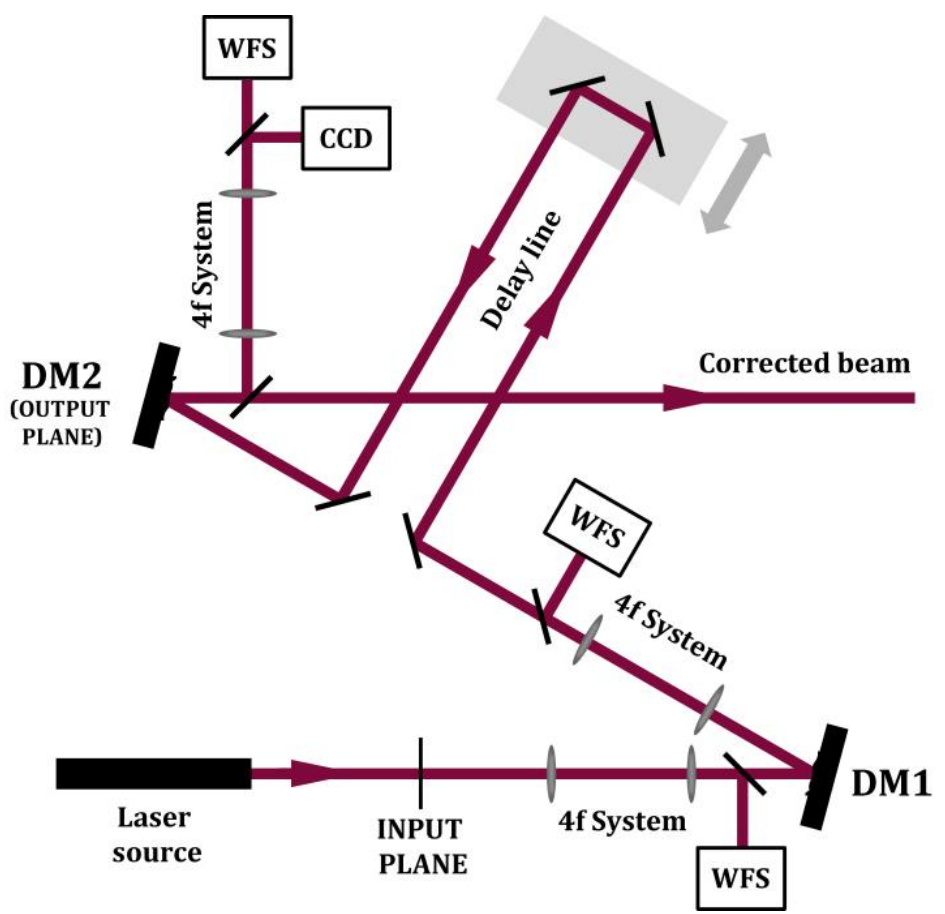

Fig.2. Schematic of the active laser beam correction system implemented in the laboratory to produce a high quality beam that meets the requirements of a particular application.

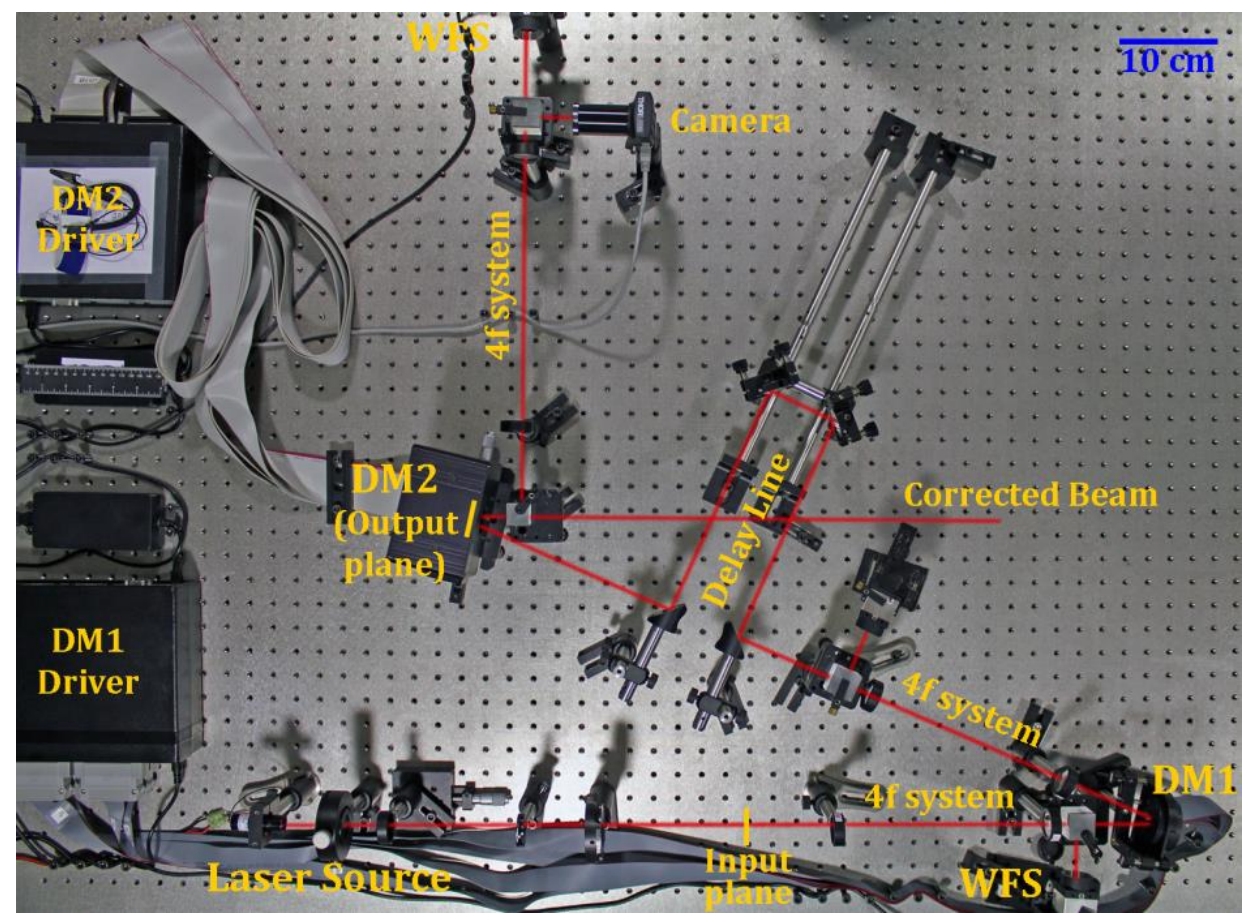

Fig.3. Photograph of the experimental set-up to obtain a phase- and amplitude-corrected beam.

The laser source is a laser diode with a power of $5 \mathrm{~mW}$ and a wavelength of $670 \mathrm{~nm}$. We employ a monochrome Thorlabs' CMOS camera (1280 x 1024 pixels with a pixel size of $5.20 \mu \mathrm{m})$ to obtain highresolution measurements of the amplitude distributions. The experimental set-up was assembled from standard optomechanical components. In Figures 2 and 3 we show the laboratory prototype where we can also see three $4 \mathrm{f}$ systems and a delay line. Each $4 \mathrm{f}$ system consists of two identical plano-convex lenses (following the light path, first two $4 \mathrm{f}$ systems use lenses with focal length of $100 \mathrm{~mm}$ and the last one uses lenses with focal length of $125 \mathrm{~mm}$ ). They are used to conjugate the WFSs with the input plane, the DMs and the output plane. The delay line allows to change the separation between the DMs. This separation is a free parameter in the algorithm (see [10]). With this arrangement we can easily modify this distance. 
The key feature in order to build an optically efficient active beam-shaping system is the minimization of the power losses. To accomplish this task, we need to remove all the optical components that could produce power losses, mainly the beam-splitters. Magnetic bases are an excellent method for mounting elements that need to be inserted and removed from the optical path with a high degree of repeatability. With this bases, all the beam-splitters and the $4 \mathrm{f}$ systems can be manually removed when the active correction is achieved.

Finally, changes in amplitude and/or phase of a laser beam could be caused by perturbations, instabilities of the laser source and/or alignment errors among others. In order to validate the algorithm with experimental data, we include a system to modify the quality of the beam at the input plane (see Fig. 2 and Fig. 3). A spatial filter usually produces a collimated beam. Physically, the spatial filter consists of a positive lens that focuses the laser beam to a small pinhole. Then, the expanded laser beam is collimated with a positive lens. We introduce different amplitude masks fabricated over diffuser plates placed between the pinhole and the collimation lens to induce perturbations on the laser beam.

\section{Numerical and Experimental results}

In this section, we present the numerical and experimental results obtained in the laboratory with the system for automatic laser beam stabilization by using two DMs (the experimental set-up defined in Section 3) and controlled by the iterative algorithm proposed by the research group. The goal is to maintain the quality of the beam (defined as a fixed desired amplitude and a fixed desired phase) at the output plane despite possible perturbations, mismatches or misalignments of the laser beam at earlier stages.

Below, we present the simulations and the experimental data obtained for two different input fields with both DMs controlled in an open-loop configuration.

\section{4.a. Laser diode}

The output beam of most semiconductor lasers is usually astigmatic and with an elliptical shape. We will use the 2-DMs concept to improve the quality of a standard diode laser. In a first step we characterize the field (amplitude and phase) at the input plane and we define the desired field (amplitude and phase) at the output plane. The desired field has been chosen as a beam with an ideal Gaussian intensity profile with FWHM $=1.5 \mathrm{~mm}$ and uniform phase. The number of iterations in the laser beam shaping algorithm was set to $\mathrm{N}=100$, and the separation between the DMs was fixed at $\mathrm{Z}=75 \mathrm{~cm}$. Figure 4 shows the input field, the desired field, the output simulated field obtained by numerical propagation and finally, the experimental field obtained in the laboratory. We display the amplitude distribution of the fields instead of the intensity distribution in order to appreciate better their shape.

The experimental characterization of the input beam confirms the elliptical shape and the presence of astigmatism in the output beam of the laser diode (input field in the active correction system). In the first row of Figure 4 we show the amplitude of the beam registered with the scientific camera and the phase obtained from the WFS described in the previous section. The phase measurement gives a Peak-to-Valley aberration (P-V) of $1.1 \mu \mathrm{m}$ and a RMS of $0.3 \mu \mathrm{m}$. The second row presents the desired output beam. The simulated output field obtained by numerical propagation (third row in Fig. 4) allows us to anticipate the limitations of the system. The amplitude presents a squared shape and the simulated output phase presents some residual astigmatism ( $\mathrm{P}-\mathrm{V}=0.4 \mu \mathrm{m}$ and a RMS of $0.1 \mu \mathrm{m}$.). However, the quality of the beam, defined as the similarity with the desired field, has increase. Finally, in the last row of the figure we present the experimental amplitude and phase obtained in the laboratory at the output plane of the active correction system. The results obtained for the output amplitude through simulations and in the experimental set-up are very close. The experimental phase is not so similar to the one predicted by the simulations but it is much flatter than the phase at the output of the laser diode. At the output plane of the active correction system, the P-V is equal to $0.5 \mu \mathrm{m}$ and the RMS is about $0.1 \mu \mathrm{m}$.

The differences between the desired field and the simulated field can be due to: the discrete representation of the fields; the modeling of the physical problem in the beam shaping algorithm and the physical limitations of the optical devices (mainly the number of actuators and the influence function of the DMs). We have checked that the influence function of the first DM is the main responsible for the bad performance in the amplitude distribution at the output plane. This was performed taking into account the influence 
function of the DM1 in the numerical propagations [15]. In this case, the maximum stroke of both DMs does not impose any limitations.
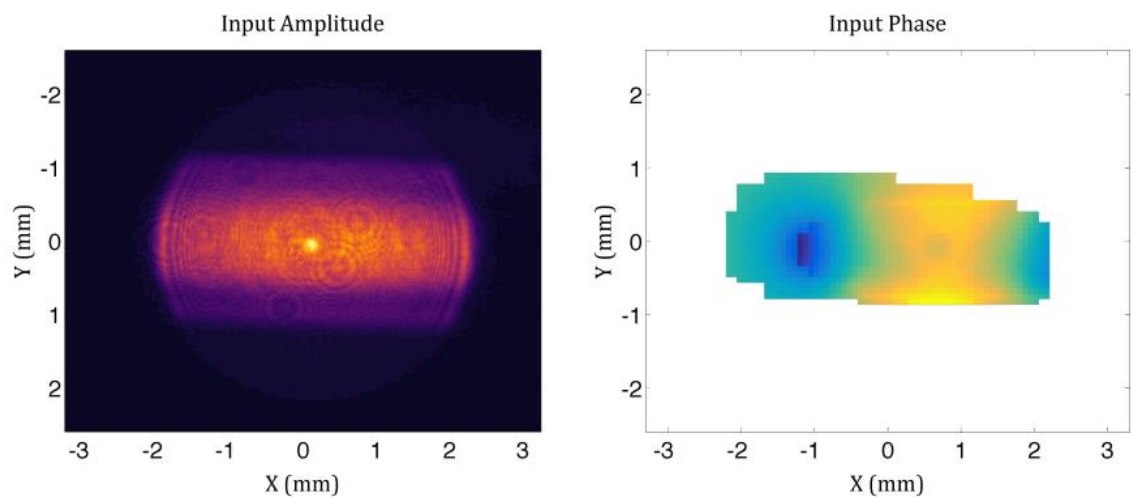

Desired Amplitude
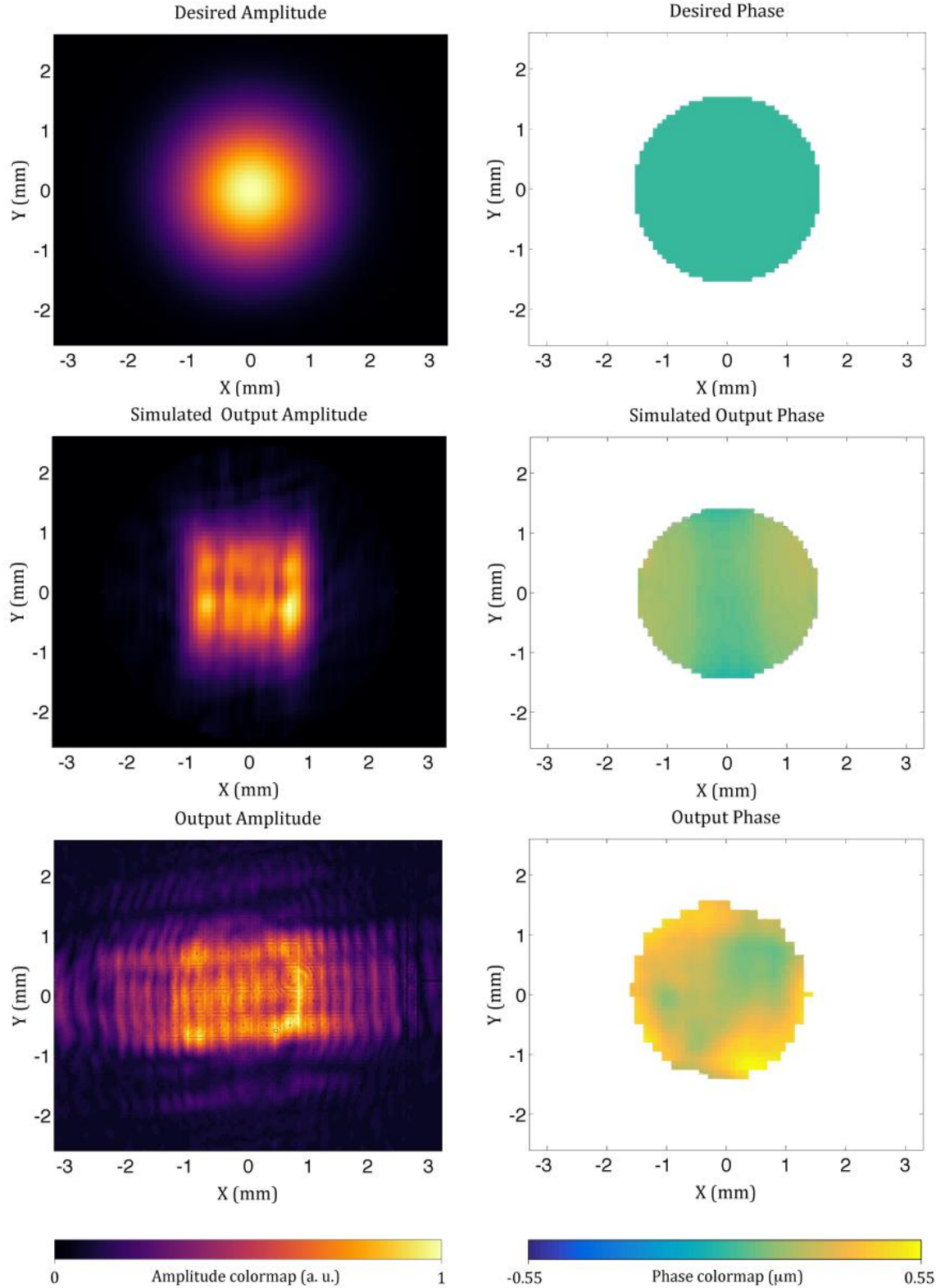

Fig.4. Experimental and numerical fields of the active correction system for the shaping case presented in section 4.a.: First row presents the input field. Second row shows the desired output field. Third row displays the results of the output field obtained by numerical propagation. Finally, in the fourth row, we present the output field registered with the experimental set-up. In the output amplitude (fourth row) the spatial pattern of the membrane of the DM2 appears in the image because the amplitude is registered in a conjugated plane of the DM2 (see Fig. 2). 
On the other hand, the differences between the simulated output field and the experimental output field can be mainly caused by: errors in the characterization and modelization of the DMs' influence function; misalignments between the beam and the position of the DMs and the WFSs; uncertainty in the determination of the input and output phases with the WFSs and diffractive effects produced by the spatial pattern on the membrane DMs (this pattern is not considered in the simulations). Controlling the DMs in a close-loop configuration could solve most of these issues. It is also possible to obtain greater control over the output phase by using the measured phase directly at the output plane instead of using the phase obtained by numerical propagation.

Although the results are not completely satisfactory, we think that the case presented in this section was very extreme due to the big amount of astigmatism and ellipticity of the input beam. By doing a vertical profile on the input amplitude (see Fig. 4), one can see that most of the energy is confined in approximately one millimeter that corresponds to only two actuators $(2 \times 450 \mu \mathrm{m})$. In spite of this, the 2-DM concept has proved to be stable and it has improved the quality of the beam.

\section{4.b. Arbitrary amplitude and constant phase distribution.}

We start by making a spatial filtering of the laser to improve the quality of the laser beam. When we achieve a high quality laser beam, we introduce an amplitude mask fabricated over a diffuser plate placed between the pinhole and the collimation lens. With this technique we mainly change the shape of the beam while the phase remains almost flat. This was verified experimentally with a WFS. Thus, in this case the input phase is always assumed flat in the algorithm. The desired field has been chosen as a beam with an ideal Gaussian intensity profile but with a smaller FWHM equal to $1 \mathrm{~mm}$ and also with a constant phase. We show in Figure 5 the same data that in Figure 4 for one of these cases.

The performance of the system is better than in the previous section. The shape of the input field is not perfectly Gaussian. However, the simulated output field is quite similar to the desired Gaussian shape and the simulated phase is almost flat $(\mathrm{P}-\mathrm{V}=0.2 \mu \mathrm{m}$; RMS $=0.05 \mu \mathrm{m})$. The experimental results confirm the simulations, both amplitudes are quite similar and the experimental phase presents results as low as a P-V $=0.3 \mu \mathrm{m}$ and $\mathrm{a}$ RMS $=0.1 \mu \mathrm{m}$.

The differences between the experimental results and the simulated results are discussed in the previous section. Furthermore, other source of discrepancies in this case comes from the assumption of a perfect constant phase for the input beam. The differences between the desired field and the simulated field also arise for the same reasons as in the previous section. Finally, for this case we can assert that the 2-DM concept has significantly improved the quality of the beam.

\section{Conclusions}

A two-deformable-mirror active correction system was implemented in the laboratory to optimize the quality of aberrated laser beams. The complete system was tested both numerically and experimentally. The tests conducted in this study validate the proposed technique. By using totally reflective continuousfacesheet DMs the light losses are minimized. The system is designed to correct quasi-static aberrations, which usually evolve at the time-scale of hours or days. Thus, when the correction was achieved, it is possible to remove all the extra optical components that could produce power losses (mainly the beamsplitters). Thus, in terms of optical efficiency, the system offers better performance when comparing with any other active laser beam shaping method.

From the experimental results, we foresee that the correction could be significantly improved by controlling the DMs in close-loop operation. This is mainly due to: small misalignments between the actuators matrix of the DMs and the WFS measurements and due to approximations in the modeling of the DMs influence function. Furthermore, the output phase may also be improved by measuring the real phase at the output plane instead of taking the phase from numerical propagation. It would also be interesting to develop a system for generating aberrations in the beam automatically.

Finally we highlight the technique not only as an optically efficient active laser correction system. The proposed method and the experimental set-up form a complete laser beam shaping system, with the possibility to choose any desired amplitude distributions. 

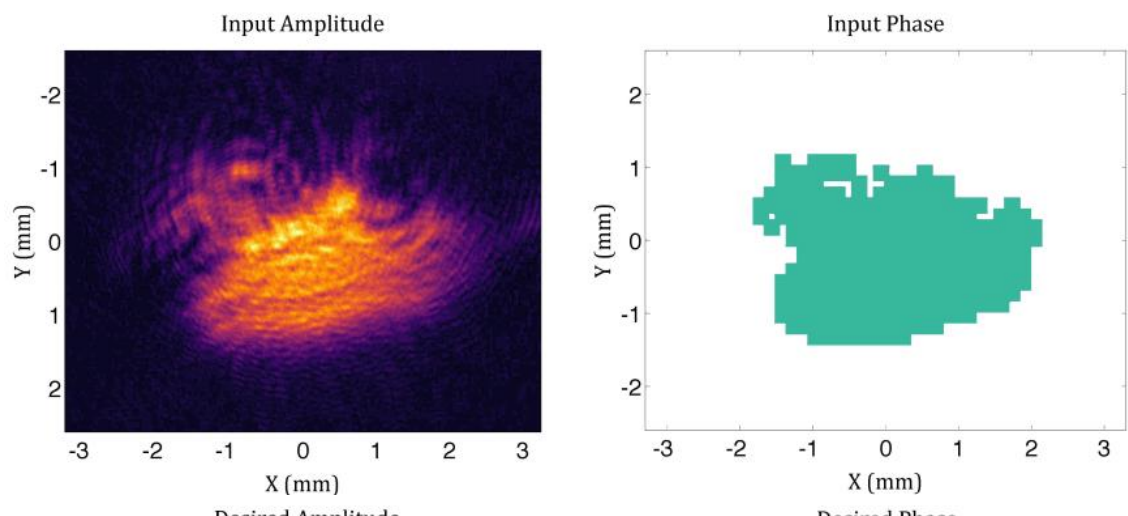

Desired Amplitude
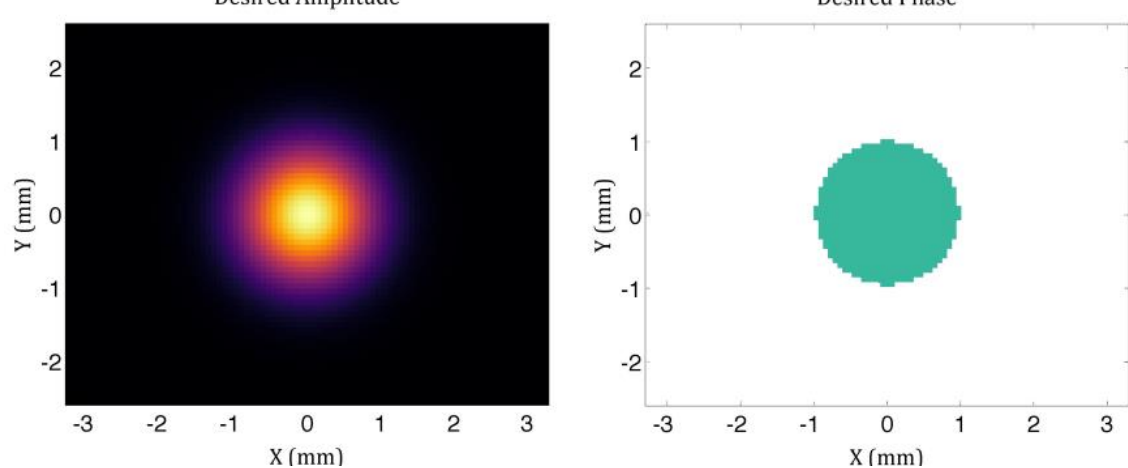

Simulated Output Amplitude
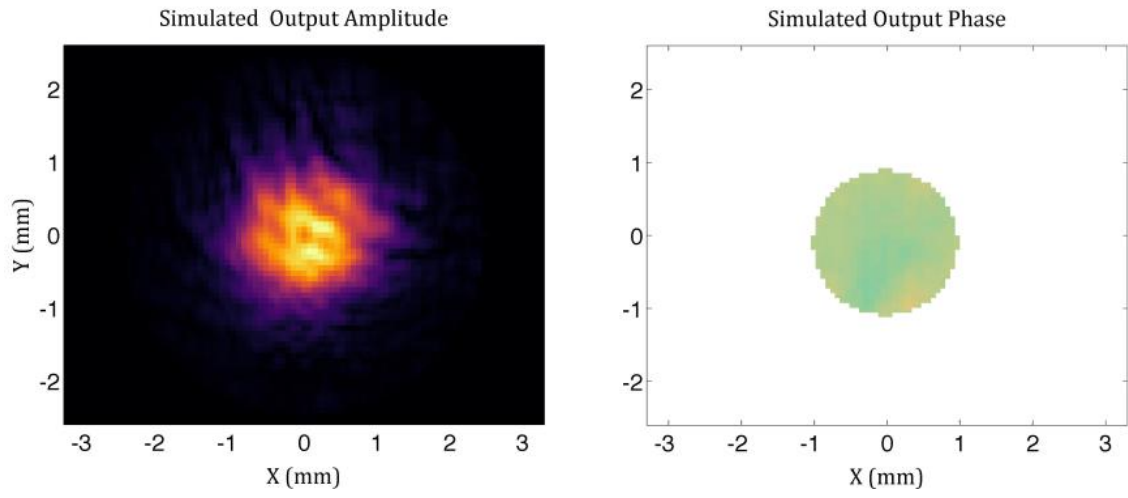

Output Amplitude
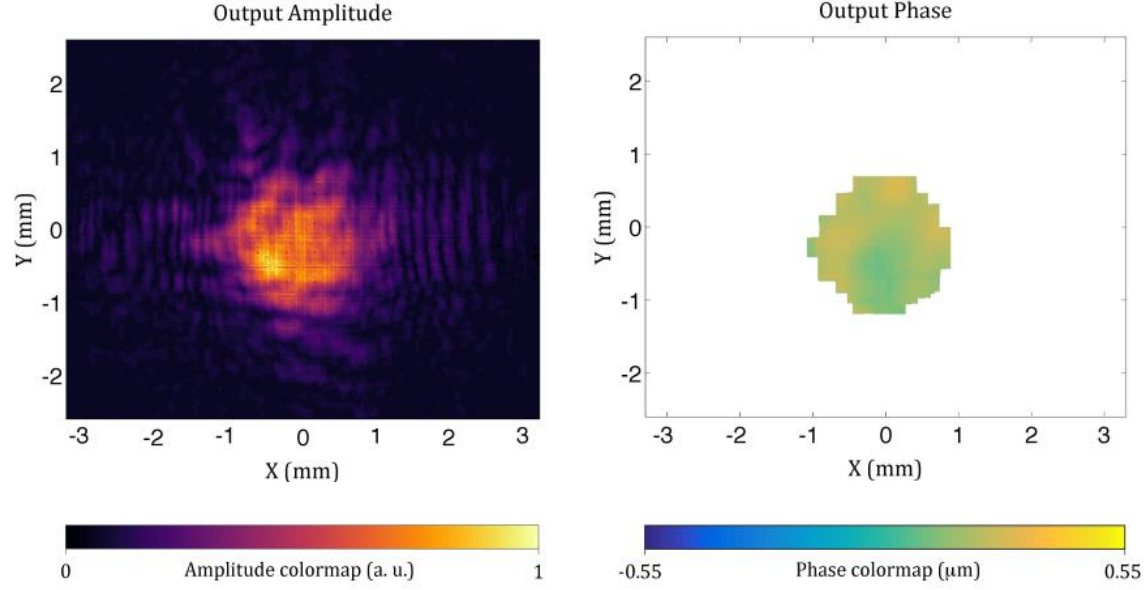

Fig.5. Same comparisons as in Fig. 4 but for the case presented in Section 4.b.

\section{Acknowledgements}

Héctor González-Núñez acknowledges the support of FONDECYT (2015 Postdoctoral Grant 3150343). Andrés Guesalaga and Clémentine Béchet thank CONICYT, grant Anillo ACT-1417. 